\title{
A Brief Look at the Examination of Kidney Biopsies under Trans- mission Electron Microscope
}

\section{Tolga Mercantepe*}

Department of Histology and Embryology, Faculty of Medicine, Recep Tayyip Erdogan University, Turkey

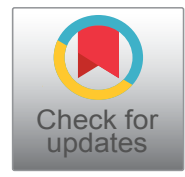

*Corresponding author: Tolga Mercantepe, Assistant Professor, Department of Histology and Embryology, Faculty of Medicine, Recep Tayyip Erdoğan University, Islampaşa Mahallesi, Iki numaralı Sehitler Caddesi, No: 74, Rize, 53010, Turkey, Tel: +90-544-255-9555, Fax: +90-464-212-30-15

Ultrastructural examination using the transmission electron microscope (TEM) has been used for diagnostic purposes in the evaluation of renal pathologies for more than 40 years $[1,2]$. Previous studies have reported that electron microscopic ultrastructural examination makes a contribution of at least $25 \%$ in the diagnosis of renal pathologies, particularly including glomerulopathies such as nephrotic syndrome and minimal change disease $[3,4]$. Several new glomerulopathies such as human immunodeficiency virus (HIV), fibrillary glomerulonephritis, membranoproliferative glomerulonephritis, and $\mathrm{C} 1 \mathrm{q}$ have been discovered in the last 25 years, and ultrastructural findings are reported to contribute to diagnosis in $85 \%$ of all renal pathologies [5] (Table 1). In a prospective study from 2011, Mokthar, et al. reported differing success rates in the diagnosis of 25 separate renal pathologies [2]. There exist various technical problems in raising this success rate in the evaluation and diagnosis of renal pathology using electron microscopic ultrastructural examination $[6,7]$. The most important of these problems derives from insufficient kidney biopsy dimensions [5-7]. The needle gauges used in the collection of renal biopsies are particularly important since these will determine the number of glomeruli in the tissue [6]. Glomerular number is of vital importance in diagnosis with renal biopsies: For example, while a single glomerulus is sufficient for diagnosis of membranous glomerulonephritis, at least seven glomeruli need to be examined for transplant diagnoses. In addition, despite the variety of diagnosis in the evaluation of renal pathology, the diagnostic success rate in renal biopsies containing 25 glomeruli is as high as 95\% [2,3,5-8]. However, 18- and 19-gauge needles are incapable of providing this amount of glomeruli $[6,9]$. Moreover, in addition to glomerulus numbers, different types of glomerulus, such as subcapsular and juxtaglomerular glomeruli are also important in diagnosis $[1,4-6,9]$. Subcapsular cortical glomeruli play a role in the diagnosis of hypertension and global sclerosis, while juxtamedullary glomeruli are reported to have an important function in the diagnosis of focal segmental glomerulosclerosis (FSGS) $[3,6,9]$. However, due to the

Table 1: Contribution of electron microscopy to diagnostic renal pathology categories.

\begin{tabular}{|l|}
\hline Acute tubular necrosis \\
\hline Alports syndrome \\
\hline Amyloid nephropathy \\
\hline Class II-VI lupus nephritis \\
\hline Combined class VI + V lupus nephritis \\
\hline Crescentic glomerulonephritis \\
\hline C1q nephropathy \\
\hline Diabetic nephropathy \\
\hline End-stage renal disease \\
\hline Fibrillary glomerulonephritis \\
\hline Focal and segmental glomerulosclerosis (FSGS) \\
\hline Hypertensive nephropathy \\
\hline IgA nephropathy and Henoch-Schonlein purpura \\
\hline IgM nephropathy \\
\hline Interstitial nephritis and pyelonephritis \\
\hline Membranous nephropathy Minimal \\
\hline Membranoproliferative types I and II \\
\hline Minimal change disease \\
\hline Post-infectious glomerulonephritis \\
\hline Thin-basement membrane \\
\hline Thrombotic mico-angiopathy \\
\hline
\end{tabular}


harmful impact on human health and the adverse effect of the fixation process, it appears unlikely that the size of biopsy specimens can be increased in the near future. For that reason, accurate identification of glomerular diseases at diagnostic renal pathology assessment requires a holistic approach involving pathological evaluation of renal biopsy with clinical data, serological tests, electron microscopy, light microscopy, and immunofluorescence (IF) [2]. Serological, immunofluorescent and light microscopic findings must be analyzed together in order to increase the success rates of diagnosing renal pathologies using the TEM. In addition, the presence of accompanying previous or correlated renal pathologies in the biopsy donor may give rise to misleading results in the diagnosis of pathologies. It is therefore important that renal pathology scales be designed by eminent and experienced pediatric and other nephrologists and histopathologists with ultrastructural experience. This will permit faster and more accurate diagnosis of renal pathologies. The design of an ergonomic multidisciplinary renal pathology diagnosis scale will increase the success of diagnosis of renal pathologies. It will also lead to new therapeutic horizons by shedding light on the damage mechanisms of diagnosed renal pathologies.

\section{Conflicts of Interest}

We declare no conflict of interest.

\section{References}

1. BH Spargo (1975) Practical use of electron microscopy for the diagnosis of glomerular disease. Hum Pathol 6: 405-420.

2. GA Mokhtar, SM Jallalah (2011) Role of electron microscopy in evaluation of native kidney biopsy: A retrospective study of 273 cases. Iran J Kidney Dis 5: 314-319.

3. S Darouich, RL Goucha, MH Jaafoura, F Ben Moussa, S Zekri, et al. (2010) Value of electron microscopy in the diagnosis of glomerular diseases. Ultrastruct Pathol 34: 49-61.

4. Y Collan, P Hirsimäki, H Aho, M Wuorela, J Sundström, et al. (2005) Value of electron microscopy in kidney biopsy diagnosis. Ultrastruct Pathol 29: 461-468.

5. A Sementilli, LA Moura, MF Franco (2004) The role of electron microscopy for the diagnosis of glomerulopathies. Sao Paulo Med J 122: 104-109.

6. AB Fogo (2003) Approach to renal biopsy. Am J Kidney Dis 42: 826-836.

7. JM Pearson, LJ McWilliam, JD Coyne, A Curry (1994) Value of electron microscopy in diagnosis of renal disease. J Clin Pathol 47: 126-128.

8. M Mubarak, J Kazi (2013) Study of nephrotic syndrome in children: Importance of light, immunoflourescence and electron microscopic observations to a correct classification of glomerulopathies. Nefrologia 33: 237-242.

9. PN Furness, S Boyd (1996) Electron microscopy and immunocytochemistry in the assessment of renal biopsy specimens: Actual and optimal practice. J Clin Pathol 49: 233-237. 